\title{
Optoretinography: optical measurements of human cone and rod photoreceptor responses to light
}

\author{
Mehdi Azimipour $^{1 *}$, Denise Valente ${ }^{1}$, Kari V. Vienola ${ }^{1}$, John S. Werner ${ }^{1}$, \\ Robert J. Zawadzki ${ }^{1,2}$, and Ravi S. Jonnal ${ }^{1}$ \\ ${ }^{1}$ Vision Science and Advanced Retinal Imaging Laboratory (VSRI), \\ Department of Ophthalmology and Vision Science, UC Davis Eye Center \\ ${ }^{2}$ EyePod Small Animal Ocular Imaging Laboratory, Department of Cell \\ Biology and Human Anatomy, University of California Davis, Davis, CA, USA \\ ${ }^{*}$ Corresponding author: mazimipour@ucdavis.edu
}

September 6, 2019

\begin{abstract}
Rods contribute crucially to human vision and their dysfunction precedes cones' in several retinal diseases. Here we describe light-evoked, functional responses of human rods and cones, measured noninvasively using adaptive optics optical coherence tomography.
\end{abstract}

Imaging the stimulus-evoked neural responses of human photoreceptors is an emerging field, with compelling potential applications in basic science, translational research, and clinical management of ophthalmic disease. It is now commonly believed that photoreceptor outer segments (OS) elongate in response to visible stimuli. This biomarker of photoreceptor function has been observed at the cellular level using common path interferometry with adaptive optics (AO) [1,2]. By providing information about the phase of the light scattered back from single cells, optical coherence tomography (OCT) has been shown capable of detecting elongations much smaller than its axial resolution [3], and this capability has been leveraged to measure light-evoked OS elongation in cones with digital aberration correction [4] and hardware AO $[5,6]$. Conventional OCT without AO has been used to observe light-evoked rod elongation in mice [7] and its converse, rod OS shortening during dark adaptation in humans [8]. Functional imaging of single human rod photoreceptors, however, has proven challenging because their small size and rapid functional response place extraordinary demands on the resolution and speed of the imaging system. At present the fastest swept-source systems have wavelengths above $1 \mu \mathrm{m}$, which provides insufficient lateral resolution for resolving rods. Alternatives such as full-field OCT may provide sufficient speed but have not yet been used to image rods.

In this work, we employed a combined AO-SLO-OCT to detect light-evoked elongation 
of rod photoreceptors. The superior lateral resolution afforded by the SLO's shorter wavelength and sub-Airy disk pinhole was used to confirm the location and type of photoreceptors in the OCT volume. The details of the combined system can be found elsewhere [9]. Briefly, the OCT system was based on a Fourier-domain mode-locked (FDML) swept-source laser with an A-scan rate of $1.64 \mathrm{MHz}$ [10]. Three 50:50 fiber couplers in a form of Michelson interferometer was used to split the light between the sample and reference arm. A balanced detector was employed to record the interference pattern and the measured sensitivity of the OCT system was $-85 \mathrm{~dB}$. The SLO images were acquired with a superluminescent diode $(\mathrm{SLD})(\lambda=840 \mathrm{~nm} ; \Delta \lambda=10 \mathrm{~nm})$. Power measured at the cornea was $1.8 \mathrm{~mW}(\mathrm{OCT})$ and $150 \mu \mathrm{W}$ (SLO), below the maximum permissible exposure (MPE) specified by ANSI [11]. The AO system was operating in a closed-loop at a rate of $10 \mathrm{~Hz}$ and by measuring and correcting aberrations over a $6.75 \mathrm{~mm}$ pupil, it provided a theoretical diffraction-limited lateral resolution of $2.75 \mu \mathrm{m}$ and $3.2 \mu \mathrm{m}$ for the SLO and OCT imaging channels, respectively.

After obtaining informed consent, two normal subjects, free of known retinal disease were imaged. Each subject's eye was dilated and cyclopleged with drops of $2.5 \%$ phenylephrine and $1 \%$ tropicamide. Subjects were dark-adapted for 30 minutes and imaged for 10-15s, with a $10 \mathrm{~ms}$ flash of $555 \mathrm{~nm}$ light delivered at $2 \mathrm{~s}$ with power of either $1 \mu \mathrm{W}, 20 \mu \mathrm{W}$, or $80 \mu \mathrm{W}$. The flashes were designed to bleach $0.2 \%, 4 \%$, and $15 \%$ of $\mathrm{L} / \mathrm{M}$-cone pigment, and $0.05 \%$, $1 \%$, and $4 \%$ of rod pigment, respectively. All procedures were in accordance with the tenets of the Declaration of Helsinki and were approved by the University of California, Davis Institutional Review Board. Acquired SLO frames were registered using a strip-based approach, and the resulting trace of eye movements was used to register the simultaneously acquired OCT volumes. Cones and rods were automatically identified in the registered images and axially segmented in the OCT volumes, providing 3D tracking of photoreceptors over time. Time-series of the complex axial signal (M-scans) of each photoreceptor were recorded and the phase difference between the IS/OS and COST and also IS/OS and ROST was measured as a function of time $[3,5]$.

In Fig. 1, panels (A) and (B) show the OCT en face image and SLO frame acquired simultaneously at $6^{\circ}$ temporal to the foveal center in a healthy subject. Clusters of rods and
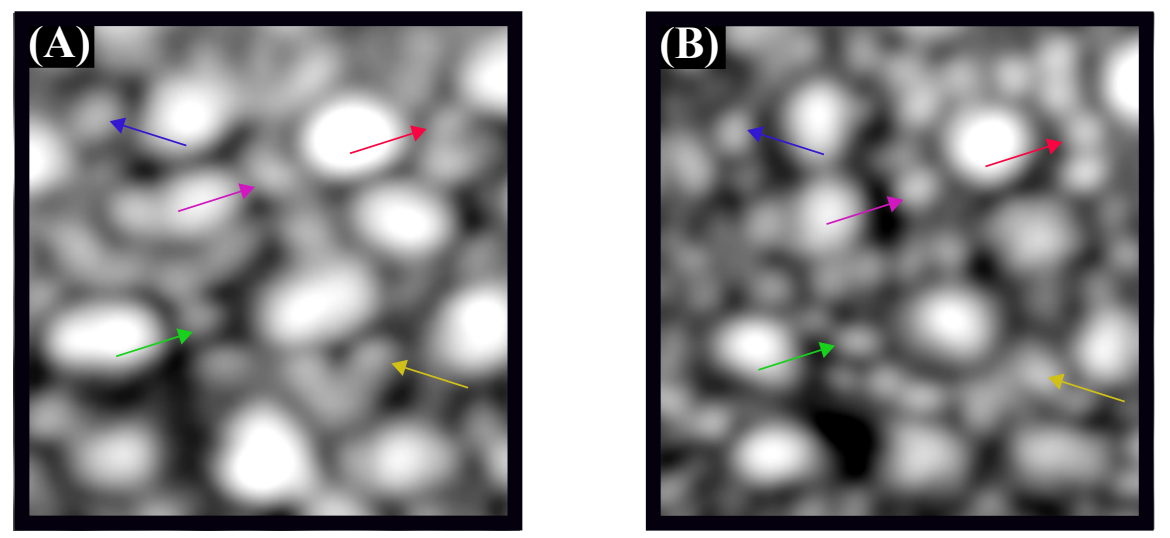

Figure 1: Example of the simultaneously acquired AO-SLO-OCT images at $6^{\circ}$ temporal to the foveal center. Rods are not as well resolved in the OCT en face projection (A) as they are in the SLO image (B). 

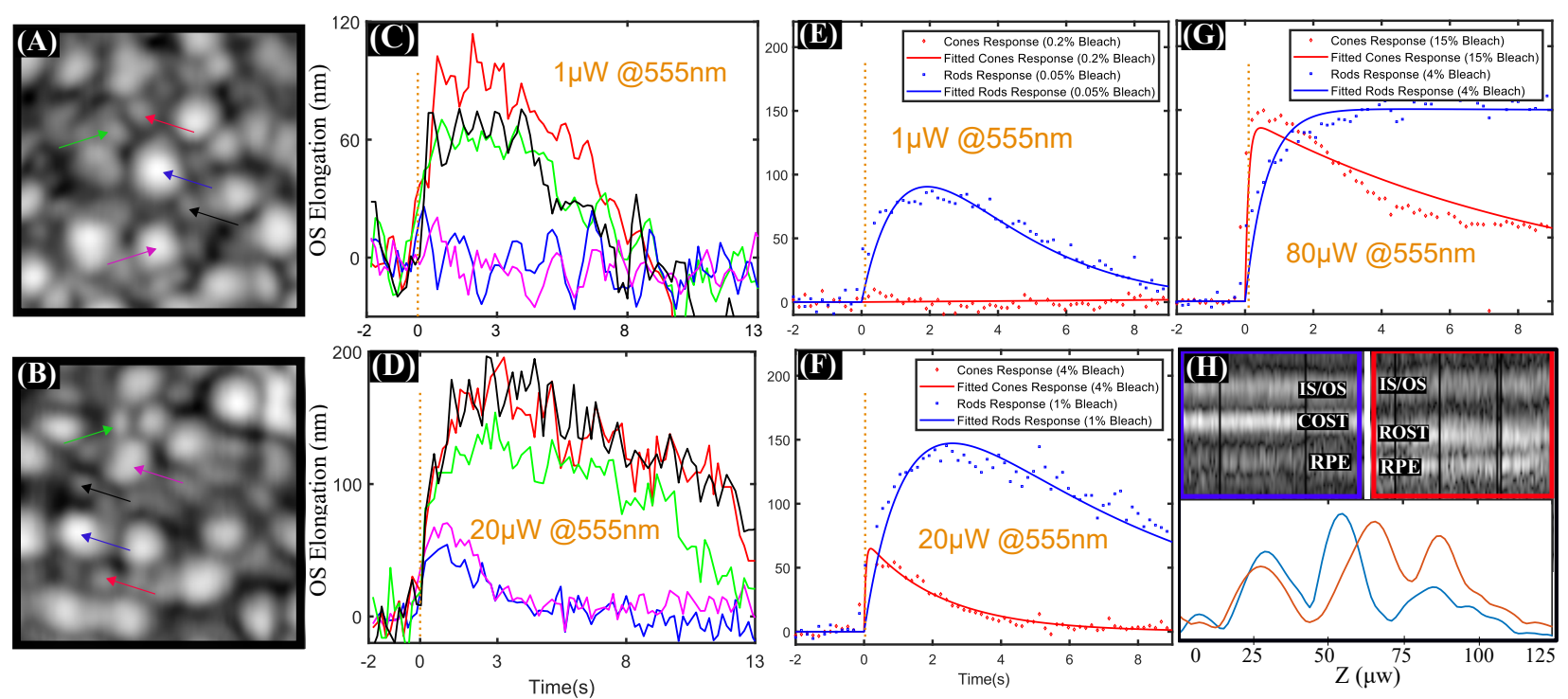

Figure 2: (A) and (B) show the OCT en face projections acquired $6^{\circ}$ temporal to the fovea. OS elongation of single rods and cones in response to $10 \mathrm{~ms}$ flashes of $1 \mu \mathrm{W}$ and $20 \mu \mathrm{W}$, at $555 \mathrm{~nm}$ are shown in panels (C) and (D), respectively. No cone response is visible in $(\mathrm{C})$. (E-G) show the averaged response of 10-20 cones and rods to $10 \mathrm{~ms}$ flashes with powers of $1 \mu \mathrm{W}, 20 \mu \mathrm{W}$ and $80 \mu \mathrm{W}$, respectively. Rod OS elongation appears to depend on flash energy but saturate at $\sim 150 \mathrm{~nm}$. (H) shows a representative M-scan of a cone (top left) and rod (top right) and their axial profiles (bottom), revealing the difference in depth of their OS tips.

some individual rods are visible in (A), distinguishable from cones by their smaller diameters. The rod clusters are revealed as individual rods in (B). The light-evoked elongation of rods and cones for three different bleaching levels are shown in Fig. 2. Panel (A) shows the OCT en face image and the locations of selected photoreceptors whose corresponding responses are shown in panel (C) for a $1 \mu \mathrm{W}$ flash. The responses of cones are not visible above the noise, but rods clearly elongate. Panels (B) and (D) show corresponding results of a $20 \mu \mathrm{W}$ flash, in which cones and rods are both observed to elongate, but with distinctive dynamics; rods exhibit a slower rate of elongation and slower recovery. Panel $(\mathrm{H})$ shows the amplitude of representative M-scans from a cone and a rod, and plots of their axial profiles, indicating their distinct axial morphology.

This work represents a powerful new tool for noninvasive measurement of both cone and rod photoreceptor function. Such measurements could represent an invaluable new biomarker in the study of retinal disease and development of cures.

Funding. R00-EY-026068 (Jonnal); R01-EY-024239 (Werner); R01-EY-026556 (Zawadzki). Acknowledgments. The authors gratefully acknowledge the assistance of Susan Garcia and additional funding from the UC Davis Eye Center and a generous donation by Dixie Henderson.

Disclosures. The authors declare no conflicts of interest. 


\section{References}

[1] Ravi S. Jonnal, Jungtae Rha, Yan Zhang, Barry Cense, Weihua Gao, and Donald T. Miller. In vivo functional imaging of human cone photoreceptors. Opt. Express, 15(24):16141-16160, 2007.

[2] Robert F Cooper, William S Tuten, Alfredo Dubra, David H Brainard, and Jessica IW Morgan. Non-invasive assessment of human cone photoreceptor function. Biomed. Opt. Express, 8(11):5098-5112, 2017.

[3] Ravi S. Jonnal, Omer P. Kocaoglu, Qiang Wang, Sangyeol Lee, and Donald T. Miller. Phase-sensitive imaging of the outer retina using optical coherence tomography and adaptive optics. Biomed. Opt. Express, 3(1):104-124, 2012.

[4] Dierck Hillmann, Hendrik Spahr, Clara Pfäffle, Helge Sudkamp, Gesa Franke, and Gereon Hüttmann. In vivo optical imaging of physiological responses to photostimulation in human photoreceptors. Proc. Natl. Acad. Sci. U.S.A., 113(46):13138-13143, 2016.

[5] Mehdi Azimipour, Justin V. Migacz, Robert J. Zawadzki, John S. Werner, and Ravi Jonnal. Functional retinal imaging using adaptive optics swept-source oct at $1.6 \mathrm{mhz}$. Optica, 6(3):300-303, 2019.

[6] Furu Zhang, Kazuhiro Kurokawa, Ayoub Lassoued, James A. Crowell, and Donald T. Miller. Cone photoreceptor classification in the living human eye from photostimulationinduced phase dynamics. Proc. Natl. Acad. Sci. U.S.A., 116(16):7951-7956, 2019.

[7] Pengfei Zhang, Robert J Zawadzki, Mayank Goswami, Phuong T Nguyen, Vladimir Yarov-Yarovoy, Marie E Burns, and Edward N Pugh. In vivo optophysiology reveals that g-protein activation triggers osmotic swelling and increased light scattering of rod photoreceptors. Proc. Natl. Acad. Sci. U.S.A., 114(14):2937-2946, 2017.

[8] Chen D Lu, ByungKun Lee, Julia Schottenhamml, Andreas Maier, Edward N Pugh, and James G Fujimoto. Photoreceptor layer thickness changes during dark adaptation observed with ultrahigh-resolution optical coherence tomography. Invest. Ophth. Vis. Sci., 58(11):4632-4643, 2017.

[9] Mehdi Azimipour, Ravi S Jonnal, John S Werner, and Robert J Zawadzki. Coextensive synchronized SLO-OCT with adaptive optics for human retinal imaging. Opt. Lett., 44(16), 2019.

[10] R. Huber, M. Wojtkowski, , and J. G. Fujimoto. Fourier domain mode locking (fdml): A new laser operating regime and applications for optical coherence tomography. Opt. Express, 14(8):3225-3237, 2006.

[11] ANSI. American National Standard for Safe Use of Lasers ANSI Z136. 1-2014. American National Standards Institute Washington, DC, 2014. 